\section{Proteins go it alone}

Two recent papers published in Nature provide the strongest evidence yet to support the protein-only hypothesis for the transmission of prion diseases. In addition, these studies - one by King and Diaz-Avalos, and the other by Tanaka and colleagues - confirm that distinct 'strains' of prion arise in the absence of genetic alterations due to differences in protein conformation.

Although it is generally accepted that proteins are the sole infectious agents in prion diseases, this has been difficult to prove. In the new studies, the authors used a yeast system to demonstrate this principle. Sup35 is a Saccharomyces cerevisiae prion that is required in normal cells for the termination of translation. Similar to the events that occur in prion diseases, Sup35 can be converted into an 'infectious' form that can propagate itself and form aggregates — known as amyloids. Cells in which this has occurred are known as $\left[\mathrm{PSI}^{+}\right]$cells, and can be distinguished from their normal counterparts, $\left[p s i^{-}\right]$cells, by alterations in their colour under certain conditions. The authors used Escherichia coli to overexpress a region of Sup35 that is sufficient to stimulate amyloid formation and purified aggregates of this protein. Expression in a bacterial system

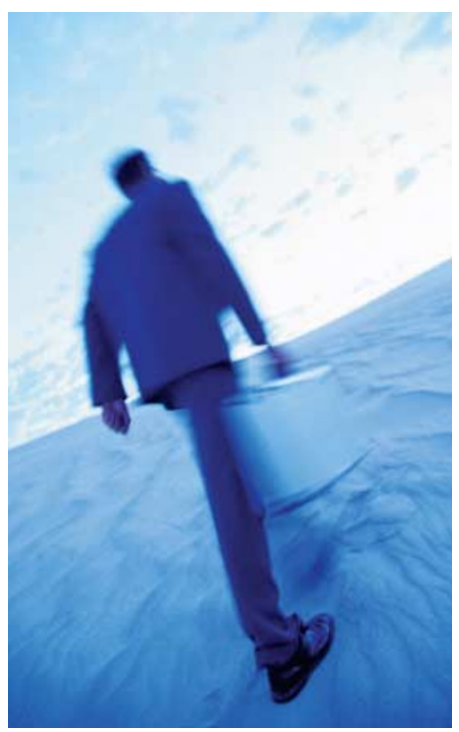

ensured the absence of any virus from the yeast cells that might be responsible for infectivity. They then used novel methods to deliver the aggregates into [psi- cells and showed that this resulted in conversion to the $\left[\mathrm{PSI}^{+}\right]$state. Protease treatment greatly decreased the infectivity of the aggregates, whereas nuclease treatment had no effect, providing the strongest evidence so far that prion proteins, in the absence of genetic material, are sufficient for infectivity.

Another contentious issue in prion research is the existence of different 'strains' of the same prion protein that vary in their infective properties. How can these distinct characteristics arise if a single protein-only agent is responsible for transmission? Both groups showed that the generation of various conformations of the same protein underlies the existence of different prion strains. In S. cerevisiae, several $\left[\mathrm{PSI}^{+}\right]$ strains can be distinguished on the basis of differential levels of Sup35 aggregation. Both groups found that when Sup35 aggregates from a specific strain were used to infect $\left[p s i^{-}\right]$ cells, the recipient cells were converted to the $\left[\mathrm{PSI}^{+}\right]$strain from which the aggregates were derived. They also showed that Sup35 amyloids with distinct conformations are generated in vitro at various temperatures and that these distinct forms induced different infective characteristics when introduced into [ $\left.p s i^{-}\right]$cells.

So, a prion protein folded in a specific way can induce the stable propagation of that conformation, with different strains being more or less efficient at converting the normal form of the protein. How this conversion takes place at the molecular level will be a key question for future investigation.

D) References and links ORIGINAL RESEARCH PAPERS King, C.-Y. \& Diaz-Avalos, R. Protein-only transmission of three yeast prion strains. Nature 428, 319-323 (2004); Tanaka, M., Chien, P., Naber, N., Cooke, R. \& Weissman, J. S. Conformational variations in an infectious protein determine prion strain differences. Nature 428, 323-327 (2004)

\section{IN BRIEF}

\section{ENVIRONMENTAL MICROBIOLOGY}

\section{Phage community dynamics in hot springs}

Breitbart, M. et al. Appl. Environ. Microbiol. 70, 1633-1640 (2004)

Breitbart et al. report the abundance of phage in extreme thermal environments (up to 1 million viruses $\mathrm{ml}^{-1}$ ) and the corresponding lysis of a large proportion of the resident bacterial species, which shows that these phage have a clear role in community dynamics. As some of these phage were robust at lower temperatures, lateral DNA transfer from these extreme environments is plausible.

\section{ANTI-INFECTIVES}

\section{Inhibition of hepatitis B virus replication by APOBEC3G}

Turelli, P. et al. Science 303, 1829 (2004)

Human APOBEC $3 \mathrm{G}$ is a cytidine deaminase that has antiretroviral activity. It is packaged into virions and deaminates deoxycytidine residues to yield deoxyuridine during viral DNA synthesis, which generates non-functional proviruses. Replication of hepatitis B virus involves reverse transcription, and expression of APOBEC3G reduced the amount of released HBV DNA by 50 -fold . This effect was reversed by supplying HIV-1 Vif protein. Vif prevents virion incorporation of the deaminase. APOBEC $3 \mathrm{G}$ catalytic activity was not required to inhibit HBV DNA replication. Instead, it seemed to block pre-genomic RNA packaging — so the mechanisms of inhibition of retroviruses and HBV by this molecule are different.

\section{BACTERIAL PATHOGENESIS}

Auto, a surface-associated autolysin of Listeria monocytogenes required for entry into eukaryotic cells and virulence

Cabanes, D. et al. Mol. Microbiol. 51, 1601-1614 (2004)

Cabanes et al. used subtractive genomics to identify Listeria monocytogenes surface proteins that were not shared with a nonpathogenic Listeria strain. They identified an autolysin (Auto) and showed that it is required for cell entry in vitro and in vivo. This new determinant for L. monocytogenes entry into eukaryotic cells is expressed independently of the master virulence-gene regulator PrfA and might function to reorganize bacterial surface architecture during infection.

\section{FUNGAL PHYSIOLOGY}

Inoculum size effect in dimorphic fungi: extracellular control of yeast-mycelium dimorphism in Ceratocystis ulmi

Hornby, J. M. et al. Appl. Environ. Microbiol. 70, 1356-1359 (2004)

Ceratocystis ulmi is a dimorphic fungus. Yeast predominate when C. ulmi is grown in vitro using a large inoculum but, with a low inoculum size, mycelia develop. This was attributed to the production of a quorum-sensing signalling molecule, which mediates the developmental switch from mycelial to yeast cell growth. 